\title{
PERFORMANCE OF SOME EGYPTIAN CLOVER CULTIVARS AND THEIR TOLERANCE TO DODDER INFESTATION
}

(Received: 17.9.2019)

\author{
By \\ Maha F. El-Enany and E. M. Zayed ${ }^{*}$ \\ Weed Research Laboratory, and ${ }^{*}$ Cell Study Research Department, Field Crops Research Institute, \\ Agricultural Research Center Giza, Egypt.
}

\begin{abstract}
Dodder parasite (Cuscuta planiflora. L) is the main obstacle which causes drastic yield losses in Egyptian forage clover crop in Egypt, accompanied with seed contamination by dodder seeds due to their similarity in size and shape to clover seeds. The present study aimed to determine the magnitude of yield losses due to dodder infestation and to estimate the degree of tolerance of some important Egyptian clover cultivars to dodder infection. Five forage clover cultivars, namely Gemmeza 1, Giza 6, Sakha 4, Helaly and Serw were tested to determine the magnitude of yield losses in these cultivars to dodder infestation and their degree of dodder tolerance under five dodder artificial infestation rates, 0 , $0.01,0.02,0.03$ and $0.04 \mathrm{~g} /$ pot of dodder seeds carried out in two pot experiments during 2015/2016 and 2016/ 2017 winter seasons in complete randomized block design. Under dodder infestation rates at $0.01,0.02,0.03$ and $0.04 \mathrm{~g} /$ pot the dry forage yield of clover losses were estimated by 32.6,37.9,47.8 and 54.4\%, respectively, in 2016 season, and 31.8,46.6,54.6 and 63.7\%,, respectively, in 2017 season as compared with the yield of clover free from dodder infestation. The effect of interaction between clover cultivars $X$ dodder seeding infestation rate (g/pot) show that studied cultivars namely Gemmeza 1, Giza 6, Helaly, and Sakha 4 had the lowest infestation rates under 0.01-0.02g/pot of dodder seeds accompanied with increases in fresh and dry weight forage /pot, stem weight and stem length and high tolerance index (70-73\%) with Sakha 4compared to El Serw 1 cultivar with the lowest tolerance index (26.1-32.9\%), and can be classified as susceptible cultivar to dodder infestation. Such results suggest that breeders can select highest tolerant cultivars of clover under artificial infestation rates accompanied with decrease in dodder infestation $\%$.
\end{abstract}

Keywords: Egyptian clover, tolerance, dodder infestation.

\section{INTRODUCTION}

Dodder (Cuscuta planiflora L.) is an obligate parasite on Egyptian clover. Dawson et al. (1994) mentioned that dodder is a completely rootle parasite free from chlorophyll and attacks the host plant for support and food supply. Khanh (2007) reported that Cuscuta spp. contains allelophathic potential exerting strong inhibition against the growth of the host plant that may be attributed to cinnamic acid and meththyl cinnamate, which are responsible for the phytotoxic action of dodder plants. Clover seeds, especially uncertified seeds are usually contaminated with huge amounts of dodder seeds as mentioned by (Lanini and Kogan, 2005; El-Refaey et al., 2014, and Abdel-Hamid and El-Khangry (2006).

El-Nahrawy et al. (2014), mentioned that resistance/tolerance to dodder among the berseem genotypes should be evaluated for fresh and dry forage yield in all cuts and seasonal yield. They found that Giza 6 had reduction of $43.3 \%$ of clover yield under dodder infestation than its healthy plants due to the reduction in chlorophyll by $94 \%$ and concluded that the reduction in fresh and dry weights of clover compared healthy clover plants are in chlorophyll reduction. Zaki et al. (1998) and Abd-El Wahed (1996) found from a histological study that dodder can attach clover stem and reach the vascular cylinder.

Dodder control requires an integrated approach to be conducted over a period of many years (Lanini and Kogan, 2005). El-Refaey et al., (2014) mentioned that uncertified clover seeds which are contaminated with dodder seeds which affecting negatively both productivity and quality of produced forage. In Egypt, dodder is a 
serious problem in the fields of forage with available clover cultivars. AL-Menofy and Hassan (1977) found that yield of fresh material and dry matter of the infested plants of berseem with Cuscuta planiflora decreased by 20 and $34 \%$ and nutritive plant materials were reduced by $31 \%$ in the infested patches as compared with healthy plants. Earliest work about the mechanism of dodder tolerance to some crop plants were done by Al- Monufi and Ashton (1991) who studied the susceptibility and resistant of some Lycopersicne species to Cuscuta campestris infection. They found that the parasite failed to grow normally in $L$. hurstum that less susceptible to dodder infestation.

Narayana and Rao (1991) studied the tolerance of blackgram and green gram varieties to dodder (Cuscuta chinensis L.) and found among fifteen blackgram and sixteen gram screened for tolerance to Cuscuta resistance that two varieties of blackgram showed some tolerance. This resistance could be due to hypersensitive reaction of the host cells to Cuscuta infestation resulting in isolation of haustorial channels from the host vascular system. There are several causes of resistance of crop plants to cuscuta infestation viz hypersensitive reaction of host cells in Lycopersicne and Phasolus spp.. (Tsivion, 1979), formation of suberized layer of cells from secondary meristem in cotton (Capderon et al., 1985).

\section{MATERIALS AND METHODS}

Two pot experiments were conducted in 2015/2016 and 2016/2017 winter seasons. Fifty $\mathrm{cm}$ pots diameter were filled with clay soil in wire house of the Weed Research Central Laboratory, Agricultural Research Center (ARC) Giza, to evaluate the effect of different infestation rates of dodder on forage yield and yield component of five Egyptian clover cultivars namely Gemmeza 1, Giza 6, Sakha 4, Helaly and El Serw1, which were obtained from the Forage Department, Field Crops Research Institute, ARC, Egypt. Each experiment consisted of 100 pots, were artificially infested with five rates of dodder seeds at 0.01, 0.02, 0.03 and $0.04 \mathrm{~g}$ dodder seeds/ pot which were collected from clover fields at Gemmeza Research Station in the previous season. Four replicates were sown in 1/10/1015 and $1 / 10 / 2016$ by the five clover cultivars. Each pot was seeded by 0.5 gram of clover seed, the forage yield as $\mathrm{g} / \mathrm{pot}$ was cut four times, every forty five days with irrigation twice each week by two liters per each pot, without fertilizers. The following data were recorded every cut.

1- Dodder infestation cover percentages per pot were determined by visual assessment.

2- Forage fresh weight, g/pot.

3- Forage dry weight, g/pot.

4- Stem length, $\mathrm{cm}$.

5- Weight of stem, g/plant.

6- Number of leaves/plant.

7- Tolerance of clover cultivars to dodder infestation by estimating tolerant index. This measure was adapted according to the scale used by Hassanein et al. (1998) as shown in Table (A). Clover yield losses for each cultivar due to dodder infestation were estimated according to the following formula.

2.1. Statistical analysis:- Collected data were statistically analyzed using analysis of variance

yield of non- infested clover (g/pot) - yield of infested clover (g/pot)

X100

$$
\text { yield of non-infested clover (g/pot) }
$$

Table (A): Suggested scale of clover tolerant to dodder infestation adopted scale used to evaluate Orobanche resistant for faba bean cultivar according to Hassanein et al., (1998).

\begin{tabular}{|c|l|}
\hline Clover crop yield tolerant index due to dodder infestation & \multicolumn{1}{|c|}{ Degree of tolerance } \\
\hline 100 & Immune \\
100 & Resistant \\
$90-70$ & Tolerant \\
60 & Moderately tolerant \\
$50-30$ & Susceptible \\
$20-0$ & Very Susceptible \\
\hline
\end{tabular}


of the split plot design according to procedure outlined by Steel and Torrie ( 1979), using MSTAT-C computer package (Freed et al. 1989). Treatments means were performed using least significant difference at $5 \%$ level probability.

\section{RESULT \&DISCUSSION}

\subsection{Effect of clover cultivars, dodder infestation seeding rate (g/pot) and interaction on dodder infestation coverage $\%$ to clover plants}

Data in Table (1) and Fig. (1) show that the effect of clover cultivars, dodder seeding rates and their interactions on dodder infestation coverage $\%$ in the four clover cuts or their general average during 2015/2016 and 2016/2017 winter seasons were statistically significant at 5 the \% level. Dodder infestation appeared in all clover cuts except with the $1^{\text {st }}$ cut in the $1^{\text {st }}$ season and the highest infestation rates appeared in the $3^{\text {rd }}$ cuts and decreased in the $4^{\text {th }}$ cut. This may be due to the variations in temperature in both seasons. Asaad et al. (1982) and El-Anany (2002) found that dodder seeds can germinate over a wide range of temperature, but the optimum temperature for seed germination as well as seedling growth is $18{ }^{\circ} \mathrm{C}$ average daily temperatures. Concerning the coverage $\%$ of dodder, it is obvious that Gemmeza 1 exert slight decrease in coverage\% in the mean of cuts with (17.3\%) than EL-Serw cultivar $(20.5 \%)$ in the $1^{s t}$ season. Meanwhile, there was no significant trend among clover cultivars except significant reduction in the coverage $\%$ in the $3^{\text {rd }}$ cut in both season, ELSerw cultivar by $(40.7 \% \& 64.7 \%)$. Increasing dodder infestation rate by dodder seeds from 0 to $0.04 \mathrm{~g}$ seeds /pot increased consistently and significantly dodder infestation coverage $\%$ on all cuts and their average were estimated at 0.04 $\mathrm{g} /$ pot rate by 37.3 and 50.1 coverage\%, in first and second seasons respectively. The effects of interactions between clover cultivars and dodder seeding rates on dodder coverage percentage were statistically significant at the 5\% level in all cuts and their general average in both seasons showed that under $0.02 \mathrm{~g}$ dodder rate infestation per pot, both Helaly \& Giza 6 followed by El Serw 1 recorded maximum reduction $\% ; 14.8 \%$ in the $1^{\text {st }}$ and $21.5 \%$ in the $2^{\text {nd }}$ season and can be described as they had some tolerance to dodder infestations and vice versa with Sakha 4 cultivar in the overall mean of the four cuts. Meanwhile, the differences between clover cultivars by dodder infestation had almost disappeared under high dodder seeding rates as shown in the average of the four cuts in the second season. These results were in agreement of those obtained by Narayana and Rao (1991) and ElNahrawy et al. (2014) in clover.

\subsection{Effect of clover cultivars, infestation rates and interaction on clover forage fresh yield in $\mathbf{g} / \mathbf{p o t}$}

Data in Table (2) and Fig. (2) showed that the effect of clover genotypes and different dodder infestation rates and their interactions on fresh clover yield in $\mathrm{g} / \mathrm{pot}$ in the four cuts and their total yield as g/pot arrived to the level of significant at the 0.05 level in both 2016and 2017 seasons. In general, with all clover cultivars and their averages the forage yield tended to decrease consistently with increasing cut number, especially in third and fourth cuts, where Helaly and Gemmeza 1 cultivars recorded the highest forage yield $\mathrm{g} /$ pot in both seasons as compared with El- Serw cultivar in all cuts averages in both seasons. Increasing dodder infestation rates caused consistent reduction in the fresh weight of clover in each cut and on their total weight in both the first and the second seasons. This may be attributed to the high dodder infestation especially in the $3^{\text {rd }}$ cut. The effects of interaction between clover cultivars X dodder seeding rate on the fresh forage yield of clover cultivars of all cuts and their average were statistically significant in all cuts expect with the first cut in both seasons. Under free dodder condition the highest forage yield was obtained from Helaly $(97.2 \mathrm{~g} / \mathrm{pot})$ followed by Giza 6 (93.3 g/pot) and lowest yield was obtained from El Serw (60.8 g/pot) in 2015/2016 season and Gemmeza 1(123.7 g/pot) and Sakha $4(112.5 \mathrm{~g} / \mathrm{pot})$ and the lowest yield by El Serw (100.4 g/pot ). These results show that these cultivars can be grown with high yielding capacity in soil seeds free from dodder infestation. Under high dodder infestation rates; $(0.04 \mathrm{~g} / \mathrm{pot})$, estimating forage yield losses of the studied cultivars under high dodder infestation of clovers as compared with the yield of free dodder infestation, the results of 2016 showed that, clover cultivars can be arranged in descending order for yield losses by Gemmeza 1 (36.2\%), Helaly (52.3\%), Sakha 4 (52.6\%), Giza $6(64.6 \%)$ and El Serw which highest yield losses\% (64.6\%), meanwhile in 2017 season Gemmeza 1 (58.12\%), Helaly (55.7\%), Sakha 4(67.3\%), Giza $6(67.2 \%)$ and El Serw which high yield losses\% (73.9\%). 


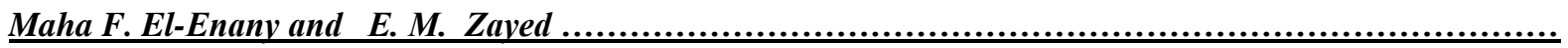

Table (1): Effect of clover cultivars and dodder infestation on dodder coverage \% which parasized on clover during 2015/2016- 2016/2017 seasons.

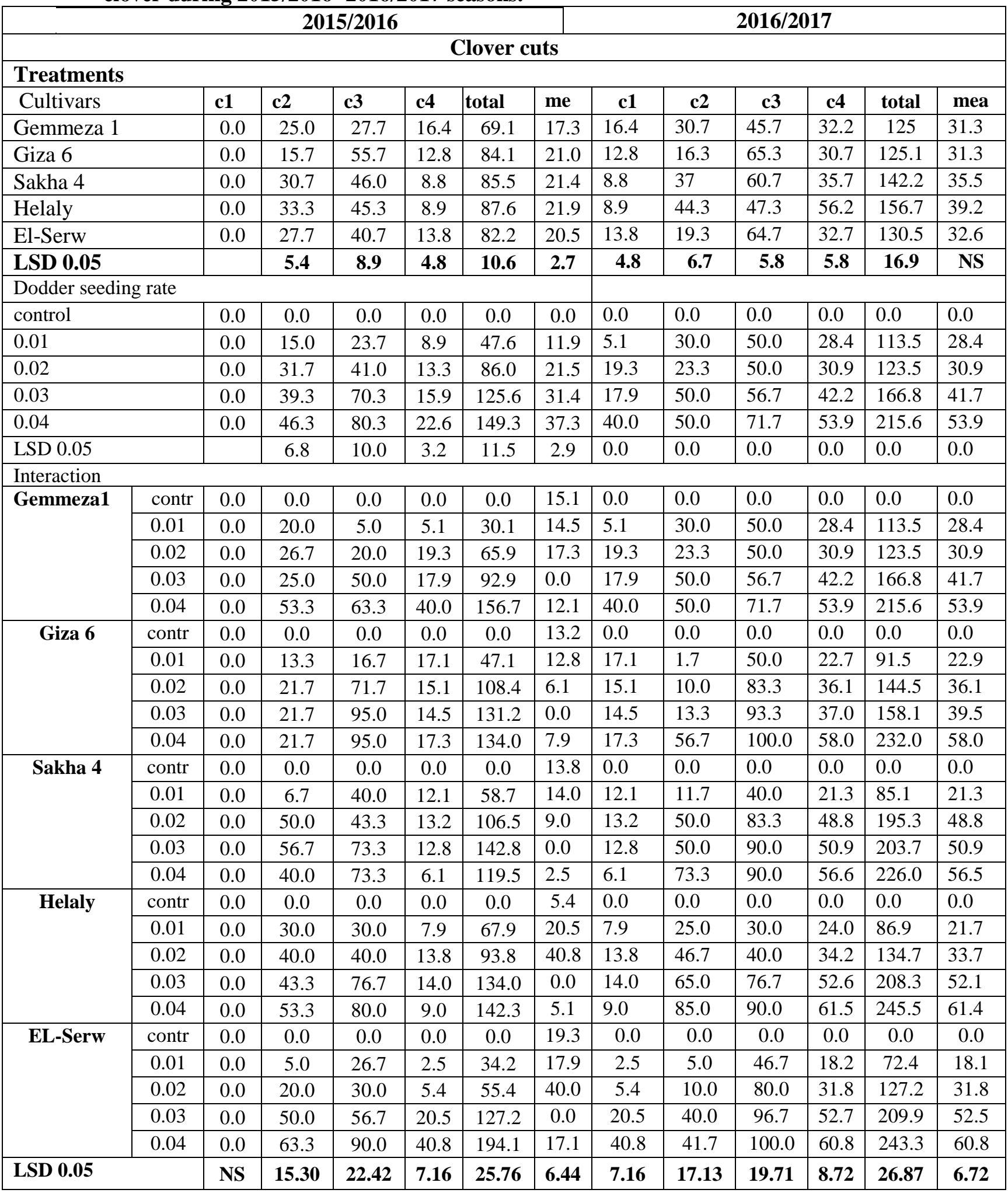

(NS not significant) 
Table (2): Effect of clover cultivars and dodder infestation rates on fresh weight of clover in g/pot during two seasons.

\begin{tabular}{|c|c|c|c|c|c|c|c|c|c|c|c|c|c|}
\hline \multicolumn{8}{|c|}{$2015 / 2016$} & \multicolumn{6}{|c|}{ 2016/2017 } \\
\hline \multicolumn{14}{|c|}{ Clover cuts } \\
\hline \multicolumn{14}{|l|}{ treatments } \\
\hline \multicolumn{2}{|l|}{ Cultivars } & c1 & $\mathbf{c 2}$ & $\mathbf{c 3}$ & c4 & total & mean & c1 & c2 & $\mathbf{c 3}$ & c4 & total & mean \\
\hline \multicolumn{2}{|l|}{ Gemmeza 1} & 103.7 & 58.7 & 47.9 & 32.1 & 242.3 & 60.6 & 112.2 & 98.8 & 46.2 & 51.9 & 309.1 & 77.3 \\
\hline \multicolumn{2}{|l|}{ Giza 6} & 87.9 & 61.0 & 41.7 & 21.3 & 212.0 & 53.0 & 105.2 & 90.5 & 39.8 & 31.3 & 266.8 & 66.7 \\
\hline \multicolumn{2}{|l|}{ Sakha 4} & 88.1 & 62.0 & 34.0 & 36.9 & 221.0 & 55.3 & 110.6 & 80.8 & 39.9 & 36.9 & 268.2 & 67.1 \\
\hline \multicolumn{2}{|l|}{ Helaly } & 93.5 & 76.8 & 64.3 & 44.4 & 279.1 & 69.8 & 108.7 & 69.6 & 43.0 & 44.4 & 265.8 & 66.5 \\
\hline \multicolumn{2}{|l|}{ El-Serw } & 45.2 & 43.9 & 24.2 & 25.2 & 138.5 & 34.6 & 106.5 & 67.6 & 33.2 & 25.2 & 232.6 & 58.1 \\
\hline \multicolumn{2}{|l|}{ LSD 0.05} & 14.1 & 12.6 & 13.0 & 14.4 & 37.2 & 9.3 & NS & 9.6 & 7.2 & 13.3 & 17.7 & 4.4 \\
\hline \multicolumn{14}{|c|}{ Dodder seeding rate } \\
\hline \multicolumn{2}{|c|}{ control } & 98.2 & 88.7 & 77.9 & 68.7 & 333.6 & 83.4 & 114.9 & 120.3 & 113.7 & 93.7 & 442.6 & 110.7 \\
\hline \multicolumn{2}{|l|}{0.01} & 82.4 & 61.1 & 44.9 & 36.1 & 224.6 & 56.2 & 110.9 & 103.9 & 46.5 & 40.8 & 302.1 & 75.5 \\
\hline \multicolumn{2}{|l|}{0.02} & 82.1 & 58.1 & 43.1 & 24.0 & 207.2 & 51.8 & 108.6 & 79.7 & 21.2 & 27.0 & 236.4 & 59.1 \\
\hline \multicolumn{2}{|l|}{0.03} & 80.3 & 46.7 & 29.3 & 19.0 & 175.3 & 43.8 & 109.6 & 61.6 & 11.8 & 17.6 & 200.6 & 50.2 \\
\hline \multicolumn{2}{|l|}{0.04} & 75.2 & 47.8 & 16.9 & 12.2 & 152.1 & 38.0 & 99.3 & 41.8 & 9.0 & 10.7 & 160.8 & 40.2 \\
\hline \multicolumn{2}{|c|}{ LSD 0.05 } & 10.3 & 8.0 & 9.8 & 11.5 & 22.5 & 5.6 & 9.6 & 9.7 & 6.9 & 11.5 & 18.7 & 4.7 \\
\hline \multicolumn{14}{|c|}{ Interaction } \\
\hline \multirow{5}{*}{ Gemmeza 1} & control & 111.4 & 74.7 & 80.0 & 55.5 & 321.6 & 80.4 & 111.2 & 133.7 & 111.0 & 138.8 & 494.7 & 123.7 \\
\hline & 0.01 & 105.2 & 56.7 & 44.2 & 29.8 & 235.8 & 59.0 & 113.4 & 116.3 & 56.3 & 59.8 & 345.8 & 86.4 \\
\hline & 0.02 & 96.7 & 57.8 & 49.6 & 30.8 & 234.9 & 58.7 & 119.5 & 104.4 & 16.7 & 30.8 & 271.5 & 67.9 \\
\hline & 0.03 & 103.8 & 49.1 & 37.0 & 24.1 & 214.0 & 53.5 & 113.8 & 74.6 & 20.9 & 17.3 & 226.7 & 56.7 \\
\hline & 0.04 & 101.2 & 55.2 & 28.5 & 20.4 & 205.3 & 51.3 & 103.2 & 64.9 & 26.2 & 12.7 & 207.0 & 51.8 \\
\hline Giza 6 & control & 92.2 & 119.7 & 92.0 & 69.4 & 373.2 & 93.3 & 108.9 & 114.0 & 110.8 & 111.2 & 444.9 & 111.2 \\
\hline & 0.01 & 87.8 & 69.6 & 69.0 & 35.4 & 261.7 & 65.4 & 100.1 & 116.6 & 48.4 & 28.7 & 293.8 & 73.5 \\
\hline & 0.02 & 84.8 & 50.0 & 41.9 & 1.7 & 178.4 & 44.6 & 105.0 & 90.2 & 27.1 & 16.7 & 239.0 & 59.8 \\
\hline & 0.03 & 86.3 & 28.3 & 0.0 & 0.0 & 114.6 & 28.6 & 103.1 & 82.8 & 12.8 & 0.0 & 198.8 & 49.7 \\
\hline & 0.04 & 88.7 & 37.6 & 5.8 & 0.0 & 132.0 & 33.0 & 108.7 & 48.8 & 0.0 & 0.0 & 157.5 & 39.4 \\
\hline Sakha 4 & control & 109.0 & 87.3 & 76.1 & 68.7 & 341.1 & 85.3 & 124.8 & 129.9 & 126.7 & 68.7 & 450.1 & 112.5 \\
\hline & 0.01 & 85.7 & 69.9 & 31.5 & 59.6 & 246.7 & 61.7 & 121.9 & 109.0 & 54.1 & 59.6 & 344.6 & 86.2 \\
\hline & 0.02 & 85.0 & 55.5 & 30.9 & 17.4 & 188.9 & 47.2 & 101.8 & 66.9 & 17.6 & 17.4 & 203.7 & 50.9 \\
\hline & 0.03 & 85.7 & 48.9 & 14.8 & 17.4 & 166.9 & 41.7 & 114.8 & 62.8 & 0.0 & 17.4 & 195.0 & 48.8 \\
\hline & 0.04 & 75.1 & 48.2 & 16.8 & 21.5 & 161.5 & 40.4 & 89.8 & 35.4 & 1.0 & 21.5 & 147.7 & 36.9 \\
\hline Helaly & control & 115.2 & 98.1 & 88.9 & 86.8 & 388.9 & 97.2 & 110.3 & 110.3 & 114.3 & 86.8 & 421.7 & 105.4 \\
\hline & 0.01 & 91.3 & 63.6 & 50.6 & 22.4 & 227.9 & 57.0 & 112.0 & 80.7 & 31.3 & 22.4 & 246.4 & 61.6 \\
\hline & 0.02 & 105.5 & 96.6 & 85.2 & 49.8 & 337.1 & 84.3 & 109.5 & 57.1 & 28.6 & 49.8 & 245.0 & 61.2 \\
\hline & 0.03 & 82.7 & 63.4 & 69.6 & 44.1 & 259.8 & 65.0 & 108.9 & 53.1 & 23.1 & 44.1 & 229.2 & 57.3 \\
\hline & 0.04 & 72.7 & 62.5 & 27.3 & 19.2 & 181.7 & 45.4 & 102.8 & 46.9 & 17.9 & 19.2 & 186.9 & 46.7 \\
\hline El-Serw & control & 63.4 & 63.8 & 52.6 & 63.2 & 243.0 & 60.8 & 119.1 & 113.8 & 105.6 & 63.2 & 401.7 & 100.4 \\
\hline & 0.01 & 42.1 & 46.1 & 29.3 & 33.5 & 151.0 & 37.7 & 106.9 & 97.0 & 42.6 & 33.5 & 280.1 & 70.0 \\
\hline & 0.02 & 38.5 & 30.4 & 7.7 & 20.1 & 96.8 & 24.2 & 107.1 & 79.7 & 15.7 & 20.1 & 222.7 & 55.7 \\
\hline & 0.03 & 43.3 & 43.8 & 25.1 & 9.2 & 121.4 & 30.4 & 107.2 & 34.8 & 2.2 & 9.2 & 153.4 & 38.4 \\
\hline & 0.04 & 38.6 & 35.4 & 6.1 & 0.0 & 80.2 & 20.0 & 92.0 & 12.9 & 0.0 & 0.0 & 104.9 & 26.2 \\
\hline LSD0.05 & & NS & 18.0 & 21.9 & 25.6 & 50.3 & 12.6 & NS & 21.7 & 15.4 & 25.6 & 41.8 & 10.4 \\
\hline
\end{tabular}

(NS not significant)

These results emphasized the fact that Gemmeza 1 followed by Helaly were more tolerant than the other clover cultivars and ElSerw can be considered as susceptible cultivar. These results are confirmed by Al- Menoufi and Hassan (1977) and El-Nahrawy et al. (2014).

\subsection{Effect of clover cultivars, dodder seeding rates and their interaction on dry weight clover forage}

Data in Table (3) and Fig. (3) showed that clover cultivars Gemmeza 1, Giza 6, Sakha 4 and Helaly significantly exceeded El-Serw 


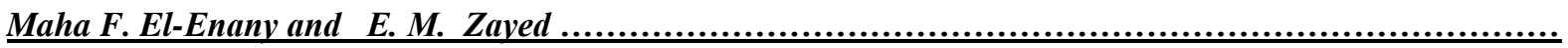

Table (3): Effect of clover cultivars, dodder seedingand interaction on dry weight of clover on yield during two seasons.

\begin{tabular}{|c|c|c|c|c|c|c|c|c|c|c|c|c|c|}
\hline & \multicolumn{7}{|c|}{$2015 / 2016$} & \multicolumn{6}{|c|}{$2016 / 2017$} \\
\hline \multicolumn{14}{|c|}{ Clover cuts } \\
\hline \multicolumn{14}{|l|}{ Treatments } \\
\hline \multicolumn{2}{|l|}{ Cultivars } & c1 & c2 & c3 & c4 & total & mean & c1 & $\mathbf{c 2}$ & c3 & c4 & total & mean \\
\hline \multicolumn{2}{|l|}{ Gemmeza 1} & 19.7 & 12.1 & 5.5 & 6.8 & 44.1 & 11.0 & 20.0 & 19.9 & 9.5 & 16.6 & 66.0 & 16.5 \\
\hline \multicolumn{2}{|l|}{ Giza 6} & 20.3 & 6.9 & 6.3 & 3.4 & 36.9 & 9.2 & 21.3 & 18.4 & 11.3 & 17.0 & 68.0 & 17.0 \\
\hline \multicolumn{2}{|l|}{ Sakha 4} & 21.1 & 11.8 & 6.5 & 6.8 & 46.2 & 11.5 & 23.0 & 16.5 & 7.8 & 15.8 & 63.1 & 15.8 \\
\hline \multicolumn{2}{|l|}{ Helaly } & 21.6 & 9.3 & 7.6 & 12.6 & 51.1 & 12.8 & 21.8 & 14.8 & 8.9 & 16.0 & 61.5 & 15.4 \\
\hline \multicolumn{2}{|l|}{ El-Serw } & 10.7 & 8.6 & 3.8 & 5.5 & 28.6 & 7.1 & 21.4 & 13.6 & 8.0 & 14.4 & 57.4 & 14.4 \\
\hline \multicolumn{2}{|l|}{ LSD 0.05} & 5.3 & 3.3 & 2.2 & 3.7 & 10.6 & 2.7 & 1.2 & 1.4 & 2.1 & 3.9 & 4.7 & 1.2 \\
\hline \multicolumn{14}{|c|}{ Dodder seeding rate } \\
\hline \multicolumn{2}{|c|}{ control } & 19.7 & 13.9 & 12.0 & 12.6 & 58.3 & 14.6 & 24.6 & 24.2 & 22.8 & 24.0 & 95.6 & 23.9 \\
\hline \multicolumn{2}{|l|}{0.0} & 18.5 & 11.2 & 6.4 & 8.0 & 44.1 & 11.0 & 21.7 & 20.8 & 12.5 & 18.3 & 73.3 & 18.3 \\
\hline \multicolumn{2}{|l|}{0.0} & 19.7 & 7.6 & 4.0 & 6.3 & 37.6 & 9.4 & 20.5 & 16.2 & 5.0 & 13.6 & 55.3 & 13.8 \\
\hline \multicolumn{2}{|l|}{0.0} & 17.3 & 7.6 & 4.2 & 5.3 & 34.4 & 8.6 & 20.5 & 12.6 & 2.7 & 12.0 & 47.8 & 12.0 \\
\hline \multicolumn{2}{|l|}{0.0} & 18.1 & 8.5 & 3.0 & 2.9 & 32.5 & 8.1 & 20.3 & 9.5 & 2.6 & 10.8 & 43.2 & 10.8 \\
\hline \multicolumn{2}{|l|}{ LSD 0.05} & NS & 2.2 & 2.1 & 2.7 & 5.0 & 1.3 & 2.0 & 1.9 & 2.2 & 2.7 & 4.1 & 1.0 \\
\hline \multicolumn{14}{|c|}{ Interaction } \\
\hline \multirow{5}{*}{ Gemmeza 1} & control & 27.1 & 16.9 & 15.5 & 9.8 & 69.3 & 17.3 & 28.7 & 26.2 & 19.3 & 25.4 & 99.6 & 24.9 \\
\hline & 0.0 & 18.6 & 11.8 & 3.3 & 6.2 & 39.9 & 10.0 & 18.6 & 23.1 & 12.0 & 17.9 & 71.6 & 17.9 \\
\hline & 0.0 & 18.1 & 9.3 & 3.4 & 7.5 & 38.2 & 9.6 & 18.1 & 20.4 & 2.8 & 13.8 & 55.1 & 13.8 \\
\hline & 0.0 & 14.8 & 10.7 & 1.1 & 4.9 & 31.5 & 7.9 & 14.8 & 16.0 & 4.6 & 11.8 & 47.2 & 11.8 \\
\hline & 0.0 & 19.9 & 11.8 & 4.4 & 5.6 & 41.7 & 10.4 & 19.9 & 13.8 & 9.0 & 14.3 & 57.0 & 14.3 \\
\hline & control & 17.2 & 9.5 & 11.6 & 11.9 & 50.2 & 12.6 & 22.3 & 22.5 & 21.0 & 21.9 & 87.7 & 21.9 \\
\hline & 0.0 & 21.2 & 7.1 & 13.0 & 4.6 & 46.0 & 11.5 & 20.8 & 22.5 & 23.3 & 22.0 & 88.6 & 22.2 \\
\hline Giza 6 & 0.0 & 20.5 & 5.5 & 4.1 & 0.4 & 30.5 & 7.6 & 21.0 & 18.2 & 8.7 & 16.0 & 63.9 & 16.0 \\
\hline & 0.0 & 20.9 & 6.6 & 0.0 & 0.0 & 27.5 & 6.9 & 20.6 & 16.3 & 3.4 & 13.4 & 53.7 & 13.4 \\
\hline & 0.0 & 21.5 & 5.9 & 3.0 & 0.0 & 30.4 & 7.6 & 21.8 & 12.8 & 0.0 & 11.5 & 46.1 & 11.5 \\
\hline & control & 21.6 & 17.4 & 15.8 & 10.9 & 65.7 & 16.4 & 26.0 & 26.2 & 25.0 & 25.7 & 102.9 & 25.7 \\
\hline & 0.0 & 20.8 & 12.1 & 3.9 & 12.4 & 49.2 & 12.3 & 25.1 & 21.9 & 10.6 & 19.2 & 76.8 & 19.2 \\
\hline Sakha 4 & 0.0 & 20.7 & 6.8 & 3.9 & 3.5 & 34.9 & 8.7 & 20.8 & 14.0 & 3.4 & 12.7 & 50.9 & 12.7 \\
\hline & 0.0 & 20.8 & 10.3 & 4.8 & 3.6 & 39.5 & 9.9 & 23.6 & 12.9 & 0.0 & 12.2 & 48.7 & 12.2 \\
\hline & 0.0 & 21.6 & 12.1 & 3.9 & 3.9 & 41.4 & 10.4 & 19.5 & 7.3 & 0.2 & 9.0 & 36.0 & 9.0 \\
\hline & control & 21.8 & 12.7 & 11.2 & 17.8 & 63.4 & 15.9 & 21.8 & 22.7 & 23.2 & 22.6 & 90.3 & 22.6 \\
\hline Helaly & 0.0 & 22.1 & 14.7 & 5.0 & 9.9 & 51.8 & 12.9 & 22.4 & 16.7 & 6.6 & 15.2 & 60.9 & 15.2 \\
\hline & 0.0 & 26.5 & 9.6 & 6.4 & 16.4 & 58.9 & 14.7 & 22.3 & 12.2 & 5.8 & 13.4 & 53.7 & 13.4 \\
\hline & 0.0 & 20.0 & 4.5 & 12.5 & 13.7 & 50.7 & 12.7 & 22.3 & 10.9 & 5.2 & 12.8 & 51.2 & 12.8 \\
\hline & 0.0 & 17.5 & 5.1 & 2.9 & 5.0 & 30.5 & 7.6 & 20.4 & 11.3 & 3.7 & 11.8 & 47.2 & 11.8 \\
\hline & control & 11.0 & 13.0 & 6.1 & 12.7 & 42.7 & 10.7 & 23.9 & 23.3 & 25.3 & 24.2 & 96.7 & 24.2 \\
\hline & 0.0 & 9.8 & 10.1 & 6.7 & 6.9 & 33.5 & 8.4 & 21.5 & 19.7 & 10.1 & 17.1 & 68.4 & 17.1 \\
\hline EL-Serw & 0.0 & 12.7 & 6.6 & 2.4 & 3.8 & 25.6 & 6.4 & 20.5 & 16.1 & 4.4 & 13.7 & 54.7 & 13.7 \\
\hline & 0.0 & 9.8 & 5.8 & 2.8 & 4.2 & 22.6 & 5.6 & 21.3 & 6.8 & 0.4 & 9.5 & 38.0 & 9.5 \\
\hline & 0.0 & 10.1 & 7.5 & 0.9 & 0.0 & 18.5 & 4.6 & 19.9 & 2.3 & 0.0 & 7.7 & 29.9 & 7.5 \\
\hline LSD 0.05 & & NS & NS & 4.7 & NS & 11.2 & 2.8 & 4.6 & 4.3 & 5.0 & NS & 9.1 & 2.3 \\
\hline
\end{tabular}

(NS not significant) 

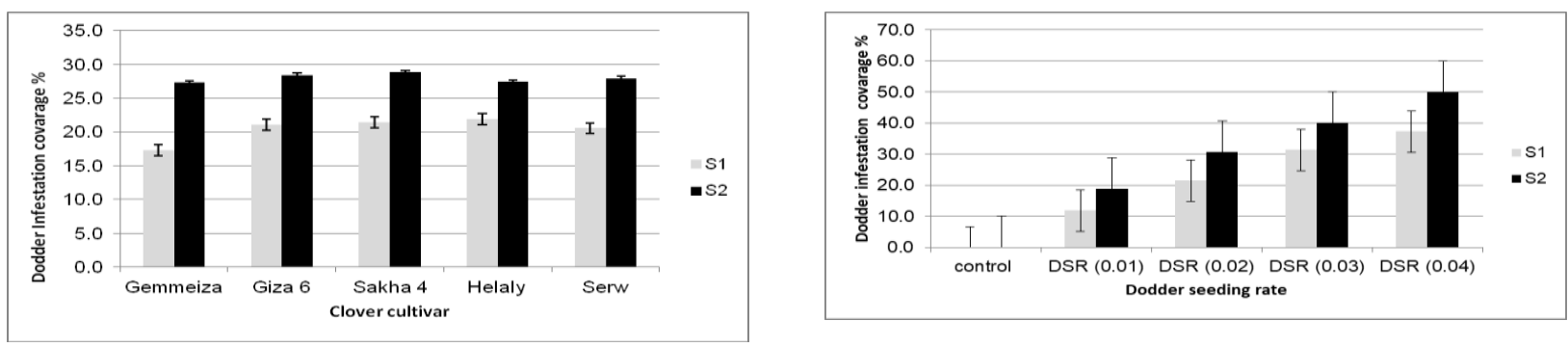

Fig. (1): Effect of clover cultivars, dodder infestation and their interaction on dodder growth during two seasons.
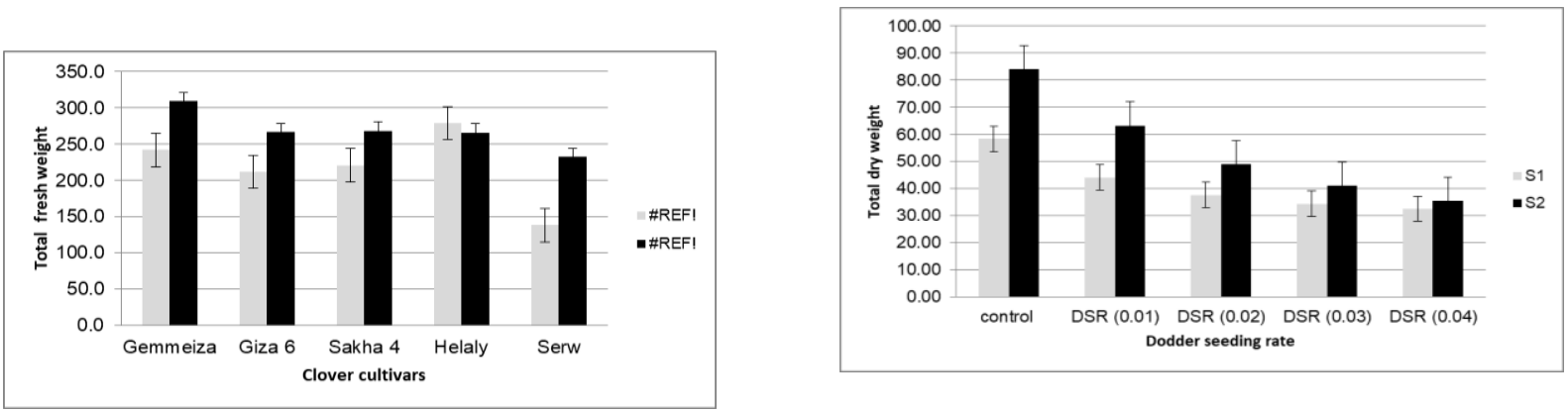

Fig.(2): Effect of clover cultivars and dodder infestation on Fresh weight during two seasons.
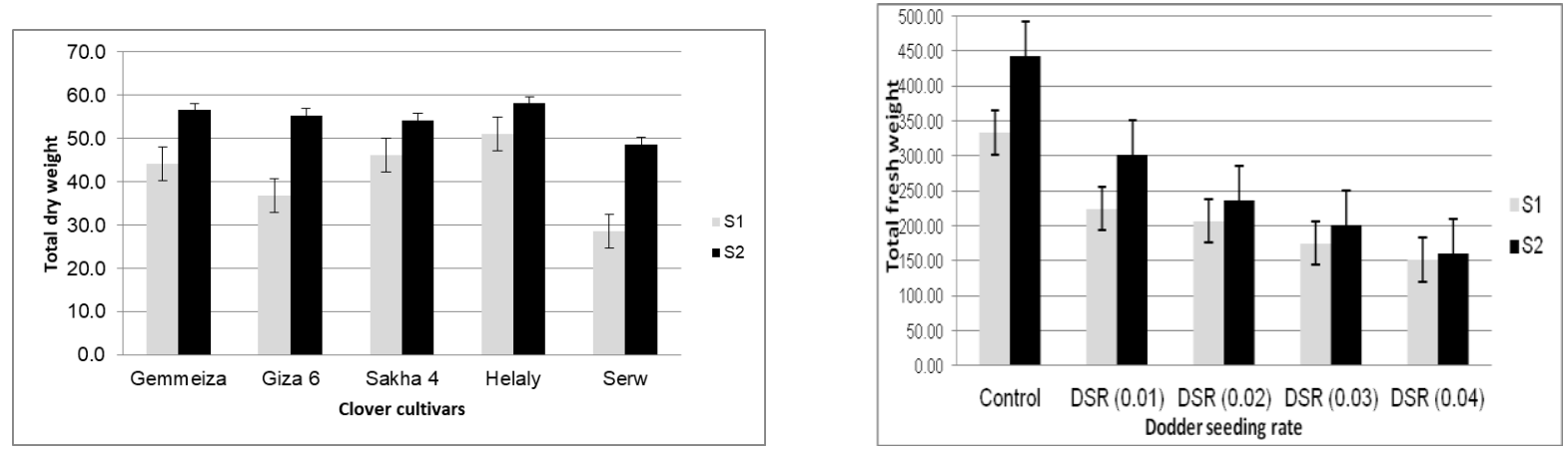

Fig. (3): Effect of clover cultivars, dodder infestation rates and their interaction on clover dry weight during two seasons. 
cultivar in their dry weight $\mathrm{g} / \mathrm{pot}$ in all cuts or their general mean with some exceptions in both $2015 / 2016$ and 2016/2017 seasons. In $2015 / 2016$, the clover cultivars can be arranged in descending order as Helaly, Sakha 4, Gemmeza 1 increased dry forage yield /pot by 80.3, 61.97and 54.93\% than El-Serw cultivar; respectively and Helaly, Gemmeza 1 by 12.9 and $15.6 \%$ than El- Serw cultivar in 2016/2017 season respectively. On the other hand, increasing dodder infestation rates from 0.01 to 0.04 gram/pot caused drastic and sharp reduction in clover dry weight than the control treatment (free dodder infestation).

The effect of the interaction between clover cultivar X seeding rates on clover dry weight of the $1^{\text {st }}$ \& the $2^{\text {nd }} \&$ the $4^{\text {th }}$ cuts in $2015 / 2016$ season and $4^{\text {th }}$ cut in 2016/2017 season did not arrive to the level of significance, meaning that the two studied factors were independent. Meanwhile, the average mean of cuts reached the level of significant in 2015/2016 season and the $1^{\text {st }} \&$ the $2^{\text {nd }} \&$ the $3^{\text {rd }}$ cuts and the average means of cuts reached to the level of significance.

Under non infestation condition the dry weight of Gemmeza 1, Giza 6, Sakha 4 and Helaly were similar in dry weight yield and exceeding significant in dry weight yield and exceeding significantly by El- Serw except with Sakha 4 in the first season and without significant differences in dry matter yield in second season, indicating that such cultivar can grow very well under non dodder infestation condition. But, under dodder infestation the day matter yield tended to decrease significantly with some variation among cultivars, with some degree of tolerance to dodder infestation except with El- Serw cultivar which is categorized as susceptible to dodder infestation. These results confirmed that obtained by Abd-El Halim et al. (1998).

\subsection{Effect of clover cultivars, dodder infestation rates and their interaction on clover stem weight $\mathbf{g} /$ plant}

The effects of clover cultivars and dodder infestation rates on clover stem weight in both studied seasons are shown in Table (4) and Fig. (4). The weight of clover plant stem as affected by clover genotypes did not reach the 5\% significant level in all cuts or their total mean in both seasons in general mean except with the first cut in the first season where Giza 6 cultivar recorded the highest stem weight per plant. The effect of various infestation rates of dodder on the weight of the stem per plant were statistically significant in all clover cuts and was true in both seasons. The trend showed that the weight of stem of clover plant tended to decline sharply with increasing dodder seeding rate from 0.01 to $0.04 \mathrm{~g}$ of dodder seeds /pot.

The effects of interaction between clover cultivar X seeding rates were not significant on stem weight per pot in all cuts except with $1^{\text {st }}$ $\& 3$ rd cuts in 2015/2016 season and $3^{\text {rd }} \& 4^{\text {th }}$ cuts in 2016/2017 season in general mean which arrived to the level of significant at 5\% level. This result pointed clearly to the susceptibility of El -Serw cultivar to dodder infestation.

\subsection{On stem length}

Table (5) and Fig. (5) showed the effect of clover cultivars, dodder seeding rates and their interaction on stem length of clover cultivars. The effect of clover cultivars were not statistically significant on stem length except in cut no 2 and no 4 in the $1^{\text {st }}$ season and all cuts in second season which arrived only to the level of significant at 5\%level. In general, the highest stem length was achieved with Gemmeza 1 and vice versa with El-Serw which recorded the lowest value of stem length.

The effect of dodder seeding infestation rates caused consistent and significant decrease on stem length with increasing infestation rates in all clover cuts or their average means in both the $1^{\text {st }}$ and $2^{\text {nd }}$ seasons. Similar results were mentioned by (Al-Menoufi\& Hassan,1977; AlMenoufi et al. 1985; Abd El-Hamid \& ElKhanagry, 2006; Goldwasser et al., 2001).

The effect of interaction between clover cultivar $\mathrm{X}$ dodder seeding rate on stem length were not statistically significant in all cuts and general mean except with $2^{\text {nd }}, 3^{\text {rd }}$ cuts and overall means which arrived to the level of significant at 5\% level in $1^{s t}$-season and from their interaction in 2016/2017 season under free dodder condition, Gimmeza 1 and Sakha 4 were significantly superior than El-Serw cultivar in stem length.

\subsection{Effect on number of leaves /plant}

The effects of clover cultivar, dodder seeding rates and their interaction on the number of leaves /clover plant in both studied seasons are shown in in Table (6) and Fig.(6). Number of clover leaves / plant did not differ significantly in both seasons as affected by clover cultivars expect with $3^{\text {rd }}$ cut in 2015/2016 and 2016/2017 which show that Helaly cultivar exerted highest mean value meanwhile, El-Serw cultivar recorded the lowest value of number of clover leaves / plants. Increasing dodder seeding rates 
Table (4): Effect of clover cultivars, dodder infestation rate and their interaction on the weight of the stem (g/pot) on dodder growth during two seasons.

\begin{tabular}{|c|c|c|c|c|c|c|c|c|c|c|c|c|}
\hline \multicolumn{7}{|c|}{$2015 / 2016$} & \multicolumn{6}{|c|}{ 2016/2017 } \\
\hline \multicolumn{13}{|c|}{ Clover cuts } \\
\hline \multicolumn{13}{|l|}{ Treatments } \\
\hline Cultivars & c1 & c2 & c3 & c4 & total & mean & c1 & c2 & c3 & c4 & total & mea \\
\hline Gemmeza 1 & 5.6 & 8 & 6.2 & 3.1 & 22.9 & 5.7 & 6.6 & 6.1 & 4.4 & 5 & 22.1 & 5.5 \\
\hline Giza 6 & 13 & 6.4 & 5.9 & 3.8 & 29.1 & 7.3 & 7.6 & 6.3 & 3.7 & 5.4 & 23 & 5.7 \\
\hline Sakha 4 & 4.7 & 6.6 & 6 & 5.4 & 22.8 & 5.7 & 6.5 & 5.6 & 5.5 & 6.5 & 24.1 & 6.0 \\
\hline Helaly & 5.1 & 6.3 & 8 & 4.7 & 24.1 & 6 & 6 & 5.4 & 4.9 & 5.8 & 22.1 & 5.5 \\
\hline El-Serw & 8.6 & 7.7 & 4.7 & 4 & 25 & 6.3 & 7.5 & 6.2 & 4.4 & 6.2 & 24.3 & 6.1 \\
\hline LSD 0.05 & 2.3 & NS & NS & NS & NS & NS & NS & $\mathbf{N S}$ & NS & NS & NS & NS \\
\hline \multicolumn{13}{|c|}{ Dodder seeding rate } \\
\hline control & 8.3 & 9.3 & 12.3 & 7 & 36.9 & 9.2 & 7.3 & 8.6 & 9.8 & 8.6 & 34.3 & 8.6 \\
\hline 0.01 & 8.9 & 8.8 & 6 & 5 & 28.7 & 7.2 & 6.8 & 7.2 & 6 & 7.3 & 27.3 & 6.8 \\
\hline 0.02 & 7.3 & 5.8 & 5.4 & 4.1 & 22.7 & 5.7 & 7.2 & 5.6 & 3.9 & 5.9 & 22.6 & 5.6 \\
\hline 0.03 & 6.8 & 6.1 & 4.1 & 2.8 & 19.7 & 4.9 & 6.5 & 4.5 & 1.8 & 4.6 & 17.4 & 4.3 \\
\hline 0.04 & 5.7 & 5 & 3.2 & 2.1 & 15.9 & 4 & 6.4 & 3.8 & 1.4 & 4.1 & 15.7 & 3.9 \\
\hline LSD 0.05 & 1.4 & 2.4 & 2.1 & 2.5 & 5 & 1.3 & NS & 1 & 1.3 & 1.8 & 2.7 & 0.7 \\
\hline
\end{tabular}

\section{Interaction}

\begin{tabular}{|c|c|c|c|c|c|c|c|c|c|c|c|c|c|}
\hline \multirow{5}{*}{ Gemmeza1 } & control & 6 & 12 & 12.4 & 3.3 & 33.7 & 8.4 & 6.6 & 7.5 & 7.2 & 7.1 & 28.4 & 7.1 \\
\hline & 0.01 & 6.7 & 10.1 & 3.2 & 2.6 & 22.5 & 5.6 & 6 & 6.1 & 5.8 & 5.4 & 23.3 & 5.8 \\
\hline & 0.02 & 7 & 5.8 & 6.7 & 3.5 & 23.1 & 5.8 & 6.8 & 6.8 & 2.8 & 7.4 & 23.8 & 6.0 \\
\hline & 0.03 & 5.3 & 6.4 & 4 & 3.8 & 19.5 & 4.9 & 7 & 5.2 & 2 & 4.7 & 18.9 & 4.7 \\
\hline & 0.04 & 3 & 5.5 & 4.9 & 2.4 & 15.8 & 4 & 6.5 & 4.7 & 4.1 & 5.1 & 20.4 & 5.1 \\
\hline \multirow{5}{*}{ Giza 6} & control & 10 & 8.5 & 11.9 & 7 & 37.4 & 9.3 & 7.4 & 9.2 & 9.5 & 8.7 & 34.8 & 8.7 \\
\hline & 0.01 & 11.3 & 6.7 & 10.3 & 8.8 & 37.1 & 9.3 & 8.3 & 7.8 & 5.6 & 7.2 & 28.9 & 7.2 \\
\hline & 0.02 & 13 & 6.2 & 6.1 & 3.2 & 28.4 & 7.1 & 8.2 & 5.7 & 2.3 & 5.4 & 21.6 & 5.4 \\
\hline & 0.03 & 14.7 & 5.5 & 0 & 0 & 20.2 & 5.1 & 7.2 & 5.3 & 1 & 4.5 & 18.0 & 4.5 \\
\hline & 0.04 & 16.2 & 4.9 & 1.4 & 0 & 22.4 & 5.6 & 7 & 3.5 & 0 & 3.5 & 14.0 & 3.5 \\
\hline \multirow{5}{*}{ Sakha 4} & control & 8 & 7.4 & 12.4 & 11.2 & 39 & 9.8 & 7.3 & 8.7 & 14.7 & 10.2 & 40.9 & 10.2 \\
\hline & 0.01 & 7 & 7.5 & 4.7 & 8.6 & 27.8 & 7 & 6.9 & 7 & 6.1 & 10 & 30.0 & 7.5 \\
\hline & 0.02 & 3 & 6.3 & 5.7 & 2.5 & 17.4 & 4.4 & 6.3 & 4.6 & 5.1 & 5.3 & 21.3 & 5.3 \\
\hline & 0.03 & 2.3 & 7.5 & 5 & 0.8 & 15.6 & 3.9 & 5.8 & 4.4 & 0 & 3.4 & 13.6 & 3.4 \\
\hline & 0.04 & 3.3 & 4.4 & 2.5 & 4.1 & 14.4 & 3.6 & 6.3 & 3.5 & 1.7 & 3.8 & 15.3 & 3.8 \\
\hline \multirow{5}{*}{ Helaly } & control & 7 & 10.1 & 14.4 & 6.1 & 37.6 & 9.4 & 6.3 & 8.7 & 7.3 & 7.4 & 29.7 & 7.4 \\
\hline & 0.01 & 6.7 & 9.1 & 4.5 & 1.1 & 21.3 & 5.3 & 6.3 & 7.6 & 5.1 & 6.3 & 25.3 & 6.3 \\
\hline & 0.02 & 5 & 4.3 & 6.8 & 7.2 & 23.3 & 5.8 & 6.2 & 4.2 & 5.2 & 5.2 & 20.8 & 5.2 \\
\hline & 0.03 & 4 & 4.9 & 8.1 & 4.9 & 22 & 5.5 & 5.6 & 3.9 & 5.7 & 6.8 & 22.0 & 5.5 \\
\hline & 0.04 & 3 & 3.1 & 6 & 4 & 16 & 4 & 5.5 & 2.7 & 1.2 & 3.1 & 12.5 & 3.1 \\
\hline \multirow{5}{*}{ El-Serw } & control & 10.7 & 8.4 & 10.3 & 7.3 & 36.6 & 9.2 & 9 & 9 & 10.3 & 9.4 & 37.7 & 9.4 \\
\hline & 0.01 & 13 & 10.8 & 7.3 & 3.8 & 34.8 & 8.7 & 6.7 & 7.4 & 7.3 & 7.1 & 28.5 & 7.1 \\
\hline & 0.02 & 8.7 & 6.5 & 1.7 & 4.3 & 21.2 & 5.3 & 8.5 & 6.8 & 3.9 & 6.3 & 25.5 & 6.4 \\
\hline & 0.03 & 7.7 & 5.9 & 3.2 & 4.5 & 21.3 & 5.3 & 6.9 & 3.5 & 0.5 & 3.5 & 14.4 & 3.6 \\
\hline & 0.04 & 3 & 7.1 & 1 & 0 & 11.1 & 2.8 & 6.5 & 4.4 & 0 & 4.3 & 15.2 & 3.8 \\
\hline \multicolumn{2}{|l|}{ LSD 0.05} & 3.1 & NS & 4.66 & NS & NS & NS & NS & NS & 3 & 4 & 6.1 & 1.5 \\
\hline
\end{tabular}

(NS not significant) 


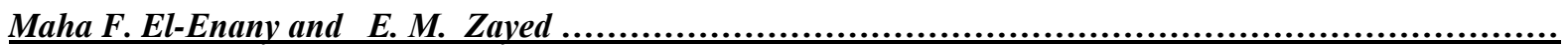

Table (5): Effect of clover cultivars and length of stem on dodder growth during two seasons. 2015/2016

2016/2017

\begin{tabular}{|c|c|c|c|c|c|c|c|c|c|c|c|c|c|}
\hline \multicolumn{14}{|c|}{$2015 / 2010$} \\
\hline \multicolumn{14}{|c|}{ Clover cuts } \\
\hline \multicolumn{14}{|l|}{ Treatments } \\
\hline \multicolumn{2}{|c|}{ Cultivars } & c1 & c2 & c3 & c4 & total & mean & c1 & c2 & c3 & c4 & total & mean \\
\hline \multicolumn{2}{|c|}{ Gemmeza 1} & 23.6 & 23.6 & 23.9 & 27.0 & 98.0 & 24.5 & 34.3 & 31.4 & 17.9 & 26.8 & 110.4 & 27.6 \\
\hline \multicolumn{2}{|l|}{ Giza 6} & 26.9 & 26.4 & 22.1 & 10.9 & 86.3 & 21.6 & 30.9 & 28.1 & 17.2 & 11.9 & 88.1 & 22.0 \\
\hline \multicolumn{2}{|l|}{ Sakha 4} & 23.2 & 22.3 & 21.6 & 24.2 & 91.3 & 22.8 & 32.6 & 29.4 & 17.9 & 24.2 & 104.1 & 26.0 \\
\hline \multicolumn{2}{|l|}{ Helaly } & 24.3 & 23.3 & 28.4 & 15.1 & 91.1 & 22.8 & 32.3 & 25.6 & 25.2 & 15.1 & 98.2 & 24.6 \\
\hline \multicolumn{2}{|l|}{ El-Serw } & 22.4 & 20.7 & 16.0 & 17.5 & 76.6 & 19.1 & 30.7 & 25.8 & 10.9 & 17.5 & 84.9 & 21.2 \\
\hline \multicolumn{2}{|l|}{ LSD 0.05} & NS & 2.2 & NS & 8.4 & NS & NS & 2.4 & 4.0 & 2.7 & 7.1 & 6.7 & 1.7 \\
\hline \multicolumn{14}{|c|}{ Dodder seeding rate } \\
\hline \multicolumn{2}{|l|}{ control } & 27.9 & 28.2 & 35.5 & 28.1 & 119.7 & 29.9 & 33.7 & 37.5 & 33.7 & 29.0 & 133.9 & 33.5 \\
\hline \multicolumn{2}{|l|}{0.01} & 27.6 & 26.5 & 26.4 & 23.1 & 103.6 & 25.9 & 32.9 & 30.8 & 21.5 & 23.9 & 109.0 & 27.3 \\
\hline \multicolumn{2}{|l|}{0.02} & 25.0 & 22.9 & 22.4 & 17.1 & 87.4 & 21.8 & 30.3 & 26.3 & 16.2 & 18.0 & 90.8 & 22.7 \\
\hline \multicolumn{2}{|l|}{0.03} & 20.9 & 20.9 & 14.5 & 15.1 & 71.4 & 17.8 & 32.4 & 23.5 & 9.6 & 13.7 & 79.1 & 19.8 \\
\hline \multicolumn{2}{|l|}{0.04} & 18.9 & 17.7 & 13.1 & 11.4 & 61.2 & 15.3 & 31.5 & 22.2 & 8.0 & 11.1 & 72.8 & 18.2 \\
\hline \multicolumn{2}{|l|}{ LSD 0.05} & 3.4 & 2.9 & 6.2 & 6.7 & 13.6 & 3.4 & 2.3 & 3.1 & 4.1 & 5.7 & 9.4 & 2.4 \\
\hline \multicolumn{14}{|l|}{ Interaction } \\
\hline \multirow[t]{5}{*}{ Gemmeza 1} & contr & 26.2 & 26.0 & 36.9 & 33.6 & 122.8 & 30.7 & 35.5 & 40.0 & 32.7 & 38.0 & 146.1 & 36.5 \\
\hline & 0.01 & 25.3 & 25.1 & 29.6 & 30.0 & 110.1 & 27.5 & 33.9 & 32.7 & 19.0 & 34.0 & 119.6 & 29.9 \\
\hline & 0.02 & 25.4 & 26.0 & 26.3 & 27.7 & 104.7 & 26.2 & 31.0 & 30.3 & 11.0 & 27.0 & 99.4 & 24.8 \\
\hline & 0.03 & 21.5 & 21.1 & 13.0 & 28.3 & 84.4 & 21.1 & 36.9 & 29.4 & 11.0 & 21.3 & 98.6 & 24.7 \\
\hline & 0.04 & 19.5 & 20.0 & 13.8 & 15.1 & 68.0 & 17.0 & 34.3 & 24.7 & 15.8 & 13.7 & 88.4 & 22.1 \\
\hline \multirow[t]{5}{*}{ Giza 6} & contr & 32.2 & 32.1 & 40.0 & 26.3 & 130.7 & 32.7 & 32.2 & 39.5 & 36.9 & 26.3 & 134.9 & 33.7 \\
\hline & 0.01 & 32.9 & 33.0 & 39.7 & 18.3 & 123.9 & 31.0 & 32.9 & 30.9 & 26.3 & 18.3 & 108.4 & 27.1 \\
\hline & 0.02 & 25.4 & 25.0 & 21.7 & 10.0 & 82.4 & 20.6 & 25.4 & 27.2 & 15.0 & 15.0 & 82.6 & 20.6 \\
\hline & 0.03 & 24.5 & 24.0 & 0.0 & 0.0 & 49.0 & 12.3 & 31.2 & 23.8 & 7.7 & 0.0 & 62.7 & 15.7 \\
\hline & 0.04 & 19.4 & 17.1 & 8.9 & 0.0 & 45.3 & 11.3 & 32.7 & 19.3 & 0.0 & 0.0 & 52.1 & 13.0 \\
\hline Sakha 4 & contr & 31.5 & 31.8 & 33.2 & 33.7 & 129.8 & 32.5 & 35.0 & 40.7 & 35.1 & 33.7 & 144.5 & 36.1 \\
\hline & 0.01 & 28.0 & 27.3 & 21.0 & 35.0 & 111.3 & 27.8 & 30.8 & 33.7 & 25.5 & 35.0 & 124.9 & 31.2 \\
\hline & 0.02 & 23.3 & 23.3 & 21.9 & 9.3 & 77.9 & 19.5 & 30.0 & 25.3 & 22.3 & 9.3 & 87.0 & 21.7 \\
\hline & 0.03 & 14.8 & 14.5 & 17.6 & 17.0 & 63.9 & 16.0 & 35.1 & 22.3 & 0.0 & 17.0 & 74.4 & 18.6 \\
\hline & 0.04 & 18.3 & 14.8 & 14.4 & 26.0 & 73.5 & 18.4 & 32.0 & 25.0 & 6.7 & 26.0 & 89.7 & 22.4 \\
\hline Helaly & contr & 27.6 & 25.3 & 39.1 & 19.7 & 111.7 & 27.9 & 33.0 & 31.7 & 37.1 & 19.7 & 121.4 & 30.4 \\
\hline & 0.01 & 29.6 & 28.5 & 24.0 & 10.3 & 92.4 & 23.1 & 33.3 & 27.3 & 19.0 & 10.3 & 90.0 & 22.5 \\
\hline & 0.02 & 26.0 & 24.4 & 29.4 & 18.3 & 98.1 & 24.5 & 35.3 & 26.5 & 26.1 & 18.3 & 106.2 & 26.6 \\
\hline & 0.03 & 19.6 & 19.1 & 25.9 & 11.7 & 76.8 & 19.2 & 30.3 & 21.5 & 26.1 & 11.7 & 89.6 & 22.4 \\
\hline & 0.04 & 18.6 & 18.0 & 23.6 & 15.7 & 76.5 & 19.1 & 29.3 & 21.1 & 17.7 & 15.7 & 83.8 & 21.0 \\
\hline El-Serw & contr & 22.3 & 25.7 & 28.2 & 27.3 & 103.5 & 25.9 & 33.0 & 35.7 & 26.5 & 27.3 & 122.5 & 30.6 \\
\hline & 0.01 & 22.1 & 18.5 & 18.0 & 21.7 & 80.2 & 20.1 & 33.3 & 29.3 & 18.0 & 21.7 & 102.3 & 25.6 \\
\hline & 0.02 & 24.8 & 16.2 & 12.6 & 20.3 & 73.9 & 18.5 & 29.7 & 22.3 & 6.8 & 20.3 & 79.1 & 19.8 \\
\hline & 0.03 & 24.0 & 24.2 & 16.1 & 18.3 & 82.8 & 20.7 & 28.3 & 20.7 & 3.1 & 18.3 & 70.4 & 17.6 \\
\hline & 0.04 & 18.7 & 18.8 & 5.0 & 0.0 & 42.5 & 10.6 & 29.0 & 21.0 & 0.0 & 0.0 & 50.0 & 12.5 \\
\hline LSD 0.05 & & NS & 6.5 & NS & NS & NS & NS & NS & NS & 9.2 & 12.7 & 21.0 & 5.3 \\
\hline
\end{tabular}

(NS not significant) 
Table (6): Effect of clover cultivars and dodder seeding rates on the number of clover per plant of leaves on dodder growth during two seasons.

\begin{tabular}{|c|c|c|c|c|c|c|c|c|c|c|c|c|}
\hline \multicolumn{7}{|c|}{$2015 / 2016$} & \multicolumn{6}{|c|}{$2016 / 2017$} \\
\hline \multicolumn{13}{|c|}{ Clover Cultivars } \\
\hline \multicolumn{13}{|l|}{ Treatments } \\
\hline Cultivars & $\mathrm{c} 1$ & $\mathrm{c} 2$ & $\mathrm{c} 3$ & $\mathrm{c} 4$ & total & mean & $\mathrm{c} 1$ & $\mathrm{c} 2$ & $\mathrm{c} 3$ & $\mathrm{c} 4$ & total & mean \\
\hline Gemmeza 1 & 32.9 & 34.4 & 20.6 & 20.9 & 108.8 & 27.2 & 32.3 & 30.7 & 20.4 & 27.7 & 111.1 & 27.8 \\
\hline Giza 6 & 34.5 & 29.9 & 19.6 & 10.5 & 94.6 & 23.7 & 37.2 & 32.1 & 20.7 & 30 & 120.0 & 30.0 \\
\hline Sakha 4 & 28.6 & 32.1 & 17.5 & 20.3 & 98.5 & 24.6 & 32.7 & 30.9 & 15.9 & 26.3 & 105.8 & 26.5 \\
\hline Helaly & 30.1 & 30.9 & 27.7 & 18.6 & 107.2 & 26.8 & 32.5 & 27.3 & 23.8 & 27.9 & 111.5 & 27.9 \\
\hline El-Serw & 29.9 & 30.5 & 17.5 & 21.3 & 99.1 & 24.8 & 30.3 & 25.3 & 11.7 & 22.4 & 89.7 & 22.4 \\
\hline LSD 0.05 & NS & NS & 2.8 & NS & NS & NS & 1.3 & 0.5 & 1.8 & NS & NS & NS \\
\hline
\end{tabular}

\begin{tabular}{|c|c|c|c|c|c|c|c|c|c|c|c|c|c|}
\hline \multicolumn{14}{|c|}{ Dodder seeding rate } \\
\hline \multicolumn{2}{|c|}{ control } & 32.9 & 34.4 & 34.2 & 33.1 & 134.7 & 33.7 & 36.4 & 36.1 & 32.5 & 35 & 140.0 & 35.0 \\
\hline \multicolumn{2}{|l|}{0.01} & 32.9 & 32.3 & 21.5 & 18.6 & 105.2 & 26.3 & 33.5 & 32.5 & 23.1 & 29.6 & 118.7 & 29.7 \\
\hline \multicolumn{2}{|l|}{0.02} & 32.7 & 30.1 & 21 & 18.5 & 102.4 & 25.6 & 33 & 28 & 17.5 & 26.6 & 105.1 & 26.3 \\
\hline \multicolumn{2}{|l|}{0.03} & 31.9 & 32.1 & 14 & 12.3 & 90.3 & 22.6 & 32.6 & 26.1 & 11.1 & 21.9 & 91.7 & 22.9 \\
\hline \multicolumn{2}{|l|}{0.04} & 25.4 & 28.8 & 12.3 & 9.1 & 75.6 & 18.9 & 29.5 & 23.5 & 8.4 & 20.4 & 81.8 & 20.5 \\
\hline \multicolumn{2}{|l|}{ LSD 0.05 } & 1.7 & 1.7 & 2.9 & 3.2 & 5.7 & 1.4 & 1.5 & 1.2 & 2.5 & 2.8 & 4.9 & 1.2 \\
\hline \multicolumn{14}{|l|}{ Interaction } \\
\hline \multirow{5}{*}{ Gemmeza 1} & control & 39.3 & 34.3 & 34.3 & 32 & 140 & 35 & 33.7 & 38 & 24.3 & 32 & 128.0 & 32.0 \\
\hline & 0.01 & 36 & 38.3 & 16.7 & 26 & 117 & 29.3 & 35.3 & 33.3 & 21.3 & 30 & 119.9 & 30.0 \\
\hline & 0.02 & 33.3 & 33.7 & 23.3 & 24 & 114.3 & 28.6 & 30.3 & 31 & 18.3 & 26.5 & 106.1 & 26.5 \\
\hline & 0.03 & 29 & 34.3 & 15.1 & 13.3 & 91.8 & 22.9 & 30.3 & 26.3 & 17.7 & 24.4 & 98.7 & 24.7 \\
\hline & 0.04 & 26.7 & 31.3 & 13.6 & 9.3 & 81 & 20.2 & 32 & 24.7 & 20.3 & 25.7 & 102.7 & 25.7 \\
\hline \multirow{5}{*}{ Giza 6} & control & 36 & 34 & 38.7 & 31.3 & 140 & 35 & 39.3 & 37.3 & 38.3 & 33.3 & 148.2 & 37.1 \\
\hline & 0.01 & 35 & 30.3 & 33.3 & 8.7 & 107.3 & 26.8 & 37.3 & 35.7 & 33.3 & 35.4 & 141.7 & 35.4 \\
\hline & 0.02 & 31 & 27.3 & 25.3 & 12.7 & 96.3 & 24.1 & 37.7 & 30 & 22 & 29.9 & 119.6 & 29.9 \\
\hline & 0.03 & 40.3 & 33 & 0 & 0 & 73.3 & 18.3 & 38 & 28.7 & 10 & 25.6 & 102.3 & 25.6 \\
\hline & 0.04 & 30.3 & 25 & 0.8 & 0 & 56.2 & 14 & 33.7 & 28.7 & 0 & 20.8 & 83.2 & 20.8 \\
\hline \multirow{5}{*}{ Sakha 4} & control & 33.7 & 36 & 29 & 36.7 & 135.3 & 33.8 & 35 & 38.7 & 34.3 & 36 & 144.0 & 36.0 \\
\hline & 0.01 & 31 & 28.3 & 15.7 & 21.9 & 96.9 & 24.2 & 32.3 & 37.3 & 20 & 30 & 119.6 & 29.9 \\
\hline & 0.02 & 32.3 & 33 & 15.7 & 8.7 & 89.7 & 22.4 & 32 & 28.7 & 19.7 & 26.6 & 107.0 & 26.8 \\
\hline & 0.03 & 27.3 & 34 & 11.3 & 12.7 & 85.3 & 21.3 & 32.7 & 27.7 & 0 & 20.1 & 80.5 & 20.1 \\
\hline & 0.04 & 18.7 & 29 & 16 & 21.7 & 85.3 & 21.3 & 31.7 & 22 & 5.7 & 19.6 & 79.0 & 19.8 \\
\hline \multirow{5}{*}{ Helaly } & control & 28.7 & 38.3 & 37.3 & 29.3 & 133.7 & 33.4 & 37.3 & 37 & 35.7 & 36.7 & 146.7 & 36.7 \\
\hline & 0.01 & 31.7 & 34.3 & 21.3 & 11.3 & 98.7 & 24.7 & 31.7 & 30 & 20.7 & 27.5 & 109.9 & 27.5 \\
\hline & 0.02 & 33.7 & 27.3 & 30.5 & 23.3 & 114.8 & 28.7 & 31.7 & 25.7 & 23.7 & 27.5 & 108.6 & 27.2 \\
\hline & 0.03 & 29.7 & 29.3 & 26.2 & 14.3 & 99.5 & 24.9 & 35 & 24 & 23 & 26 & 108.0 & 27.0 \\
\hline & 0.04 & 26.7 & 25 & 23 & 14.7 & 89.3 & 22.3 & 26.7 & 19.7 & 16 & 20.8 & 83.2 & 20.8 \\
\hline \multirow{5}{*}{ El-Serw } & control & 27 & 29.3 & 31.7 & 36.3 & 124.3 & 31.1 & 36.7 & 29.3 & 30 & 32 & 128.0 & 32.0 \\
\hline & 0.01 & 31 & 30 & 20.3 & 25 & 106.3 & 26.6 & 31 & 26 & 20.3 & 25.7 & 103.0 & 25.8 \\
\hline & 0.02 & 33.4 & 29.3 & 10 & 24 & 96.7 & 24.2 & 33.4 & 24.7 & 3.7 & 20.6 & 82.4 & 20.6 \\
\hline & 0.03 & 33.3 & 30 & 17.3 & 21 & 101.7 & 25.4 & 27 & 24 & 4.7 & 18.6 & 74.3 & 18.6 \\
\hline & 0.04 & 24.7 & 33.7 & 8 & 0 & 66.3 & 16.6 & 23.3 & 22.7 & 0 & 15.3 & 61.3 & 15.3 \\
\hline LSD 0.05 & & NS & NS & 6.4 & NS & NS & NS & NS & NS & 5.5 & 6.3 & NS & NS \\
\hline
\end{tabular}

(NS not significant) 

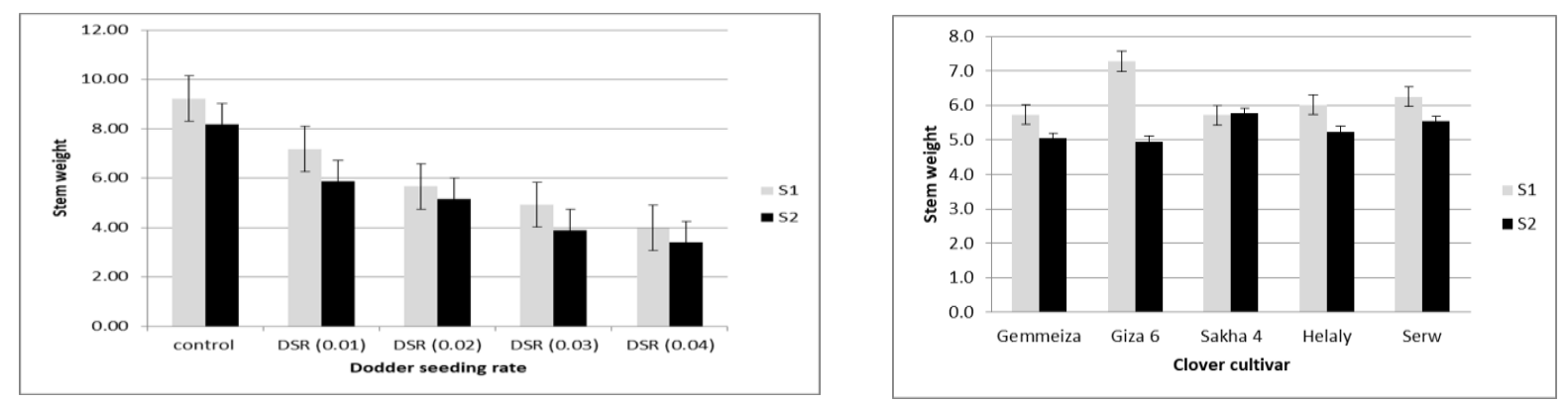

Fig. (4): Effect of clover cultivars and the weight of stem on dodder growth during two seasons.
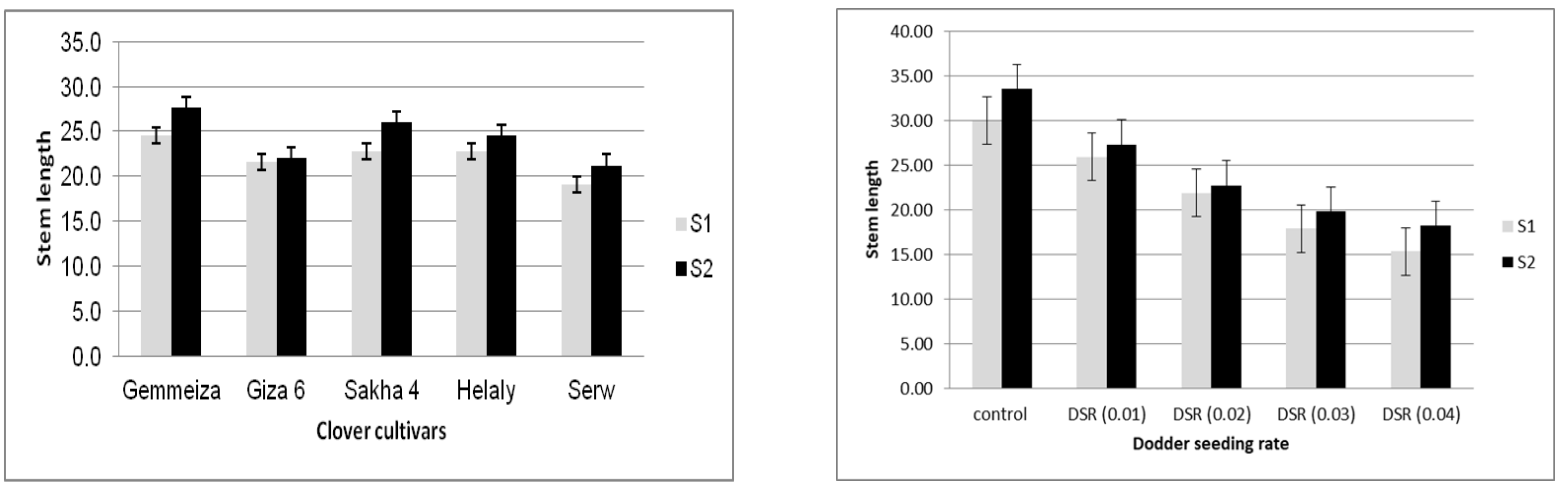

Fig. (5): Effect of clover cultivars and the length of stem on dodder growth during two seasons.
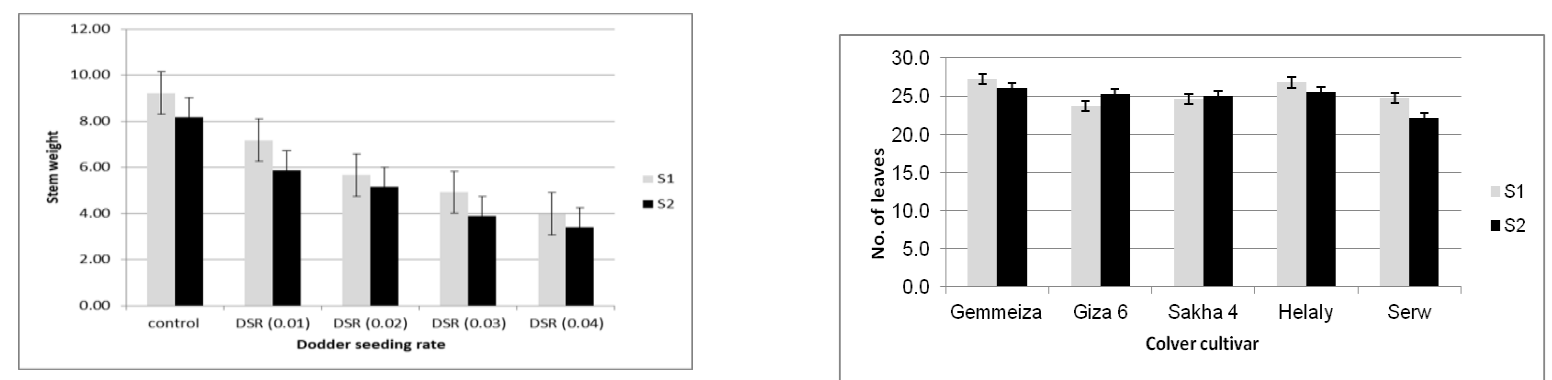

Fig. (6): Effect of clover cultivars and no. of leaves on dodder growth during two seasons. 
caused consistent and significant decreases in number of clover leaves/plant. These results were true in all cuts and average of cuts in both studied seasons, which are mainly attributed to the decrease of chlorophyll contents mentioned by (El-Nahrawy, 2014). Significant interactions were noticed between the effect of clover cultivars $\mathrm{X}$ dodder seeding rates on number clover leaves /plant on the general average, meaning that the two studied factors act independent.

\subsection{Estimation of clover cultivars tolerance to dodder infestation}

Results in Table (7) showed that Gemmeza 1 can tolerate light and medium infestation rates of dodder which had high tolerant index and almost varied from $70-73 \%$ in both seasons, while Sakha 4 had tolerant index varied from 70-
Hassanein et al., (1998) to measure the tolerance of faba bean to Orobanchae can be used to measure tolerant index of clover to dodder infestation can be used successfully as rapid technique to screen tolerant clover cultivar to dodder under artificial dodder infestation condition in pot experiments to avoid non homogeneous dodder infestation under field conditions.

\section{Conclusion}

The results of this investigation clearly showed that dodder parasitism in clover is considered as one of major biotic stresses which decrease clover forage yield by $54.4-63.7 \%$ depending on clover cultivar tolerance or the level of dodder infestation. For example Gemmeza 1 and Giza 6 tolerance indices were 73.4 and $72.3 \%$.

Table (7): Dodder index tolerance \% of clover cultivars to dodder infestation under various dodder infestation levels as average of four cuts during2015/2016-2016/2017seasons.

\begin{tabular}{|c|c|c|c|c|c|}
\hline \multirow[t]{2}{*}{ Clover cultivar Cut } & \multicolumn{4}{|c|}{ Dodder seeding rate infestation level (g/pot) } & \multirow[b]{2}{*}{0.04} \\
\hline & $\mathbf{0}$ & 0.01 & $\mathbf{0 . 0 2}$ & $\mathbf{0 . 0 3}$ & \\
\hline \multicolumn{6}{|c|}{ Tolerance\% } \\
\hline \multicolumn{6}{|c|}{$2015 / 2016$} \\
\hline Gemmeza 1 & 100 & 73.4 & 73 & 66.5 & 63.8 \\
\hline Giza 6 & 100 & 70 & 47.8 & 30.6 & 35.4 \\
\hline Sakha4 & 100 & 72.3 & 55.3 & 48.5 & 47.4 \\
\hline Helaly & 100 & 58.6 & 41.2 & 66.9 & 46.9 \\
\hline Serw & 100 & 62 & 39.8 & 50 & 32.9 \\
\hline \multicolumn{6}{|c|}{$2016 / 2017$} \\
\hline Gemmeza 1 & 100 & 69.8 & 54.9 & 45.8 & 41.9 \\
\hline Giza 6 & 100 & 66.1 & 53.8 & 44.7 & 36 \\
\hline Sakha4 & 100 & 76.2 & 45.3 & 44.4 & 32.7 \\
\hline Helaly & 100 & 58.5 & 58.1 & 53.4 & 44.4 \\
\hline El-Serw & 100 & 69.4 & 55.5 & 38.2 & 26.1 \\
\hline
\end{tabular}

100: Immune, 100: Resistant, 90-70: Tolerant, 60: Moderately tolerant, 50-30: Susceptible and 20-0: Very Susceptible.

$76.2 \%$ under light dodder seeding rate $(0.01 \mathrm{~g} / \mathrm{pot})$. Such tolerance in clover cultivars may be explained by different mechanisms as mentioned by Abd El Wahed (1996) and Zaki et al., (1998) and El Refaey et al , (2014). They state that the reaction of dodder with clover stem of tolerant clover cultivar had darkness in the tissue which stop dodder haustoria penetration in the stem and contrary to susceptible clover genotypes where in trues cells of dodder can penetrate easily to center and host vascular tissue, meanwhile Serw cultivar had low tolerant index tolerant (26.1 to 32.9\%). Thus, the technique which adopted from

\section{REFERENCE}

Abdel Halim A. Z., Oushy T.S., Sarhan G. M., El-Nohrawy M. A., Haggag M. E. and Mekhaiel G. S. (1998). Genotypic potential of some Egyptian clover population on forage yield and quality under different environmental condition. Egypt .J. Appl. Sci., 13(3) : 494 -508.

Abd El-Hamid M.M. and El-Khangry S.S. (2006). Studies on dodder Cuscuta spp. infestation in clover $T$. alexandrinum $\mathrm{L}$. fields in some governorates in Nile Delta. Egypt. J. Agric. Res., 84 (1):275-285. 
Abd El-Wahed R. (1996). Studies on the effect of certain herbicides of dodder C.spp control in certain leguminous crops. Ph.D Thesis, Fac. Agric. Cairo Univ.

Al-Menoufi O.A. and Hassan M. (1977). Studies on the parasitism of Cuscuta spp. Series 2. The effect of dodder Cuscuta planiflora Ten. on the production and chemical composition of Egyptian clover (Trifolium alexandrinum L.) Alex. J. Agric. Res. 25 (2):337-34.

Al-Menoufi O.A. and Ashfon F. M. (1991). Studies on the parasitism of Cuscuta spp. Series 8: susceptibility and resistance of some Lycoperscon species to Cuscuta campestris infection.in:proc. $5^{\text {th }}$ internetioal on parasitic weed, Nairobi, Kenya. Jun, $24^{\text {th }}-30^{\text {th }}$ pp. 293-297.

Al-Menoufi O.A., Rezk M.A., Mostafa A.K. and El- Shair S. (1985). Studies on the parasite Cuscuta spp. 6- losses in Egyptian clover and flax due to dodder infection. J. Agric. Sci. Mansoura Univ., 10(4):1262-1266.

Asaad M., Farag F.H. and Hassanein E.E. (1982).Some factors affecting dodder (Cuscuta spp.) parasitism on berseem and alfalf. Proc. Egypt. Bot. Soc. 3. (Mansura Conf.) 644-656.

Capderon M., Fer M.A. and Ozenda P. (1985). Sur un systeme inditde rejeet d'un parasite:example de la Cuscuta sur cottonnier (C. lupuliformis Krock. Sur Gossypium hirsutum L. Comptes rendu de "Academic des Scrence, $t t^{\text {" (Science de }}$ lavie., 300(6):227-237.

Dawson J.H., Musselmam L.J., Wolswinkel P. and Dörr I. (1994). Biology and Control of Cuscuta. Rev. Weed Sci., 6: 265-317.

El-Anany Maha F. (2002). Studies on Genus Cuscuta in Egypt. M.Sc. Thesis in Botany Helwan Univ.

El-Nahrawy Shereen M., El-Refaey R.A. and El Sady E.H. (2014). anatomical mechanisms of resistance/tolerance of Egyptian clover to dodder ( Cusuta spp.) Egyptian clover (Trifolium alexadrium: King of forage crops, FAO, Dost Muhammad, Bimal Misri, EL-Nahrawy, Sartaj Khan Ates Serkan ed.PP 48-52.

El-Refaey R. A., Seidy E. H., Soliman I. E.,
Fouad Samira A. and El-Nahrawy Shereen M. (2014) . Histological studies on some Egyptian clover genotype attached with dodder (Cuscuta spp.) Egypt. J. Plant Product, 5(7):1115-1128.

Freed R.S.P., Einensmith S., Gutez S., Reicosky D., Smail V.W. and Wolberg P. (1989). MSTAT.C analysis of agronomic research experamints. Michigan Univ. East Lansing, USA.

Goldwasser Y., Worobel R.L. and Lanini W. W.T. (2001). Tolerance of tomato verieties to lespedeza dodder. Weed Sci. 49:520-523

Hassanein E.E., Al Marsafy H. T., Kholosy A.S. and Ibrahim H. M. (1998). Variation in faba bean cultivars to Orobanche infection as measured by different reaction scales. Annales Agric. Sci. Ain Shams University 43.(18):189-200.

Khanh T.D., Xuan T. D. and Chung I. M. (2007). Rice allelopathy and the possibility for weed management. Annals Appl. Biol., 151(3):325-339.

Lanini, W.T. and Kogan M. (2005). Biology and management of Cuscuta in crops . Ciencia E Inestigacion Agraria, .32 (3): 165-179.

Narayna K.R. and Rao R.S.N. (1991). Studies on tolerance of black gram varieties to dodder (Cuscuta chinensis Lawn). $5^{\text {th }}$ Int. Symp. on parasitic weeds, Nairobi, Kenya June $24^{\text {th }}-30^{\text {th }} \mathrm{pp} 170-175$.

Steel R.G.D. and Torrie G.H. (1979). Principles and Procedures of Statistics. (With special Reference to the Biological Sciences.) McGraw-Hill Book Company, New York, Toronto, London 1960, 481 S., 15 Abb.; $81 \mathrm{~s} 6 \mathrm{~d}$

Tsivion Y. (1979). Morphogenitc sequences in the formation of the haustorium of Cuscuta campestris .pp 174-181.in Musselmam, L.J.;Worslmam and Eplee L.E.editors. Proc. And Int.Symp. no parasitic WWeeds. Raleigh. North Carolina State University.

Zaki M. A., El-Metwaly H.S., Hassan R.A. and Maillet J. (1998). Studies on dodder Cuscuta spp. Control. Comptes-rendus 6eme sym., Mediterranean EWRS, Montpellier, France, 13-15 Mai, 147-150. 
أداء بعض أصناف البرسيم المصرى كمحصول علف ومدى تحملها الأصابة بالحامول

مها فهيم العنانى - ايهاب محمد زاياث

المعمل المركزى لبحوث الحشائش ـ *قسم الخلية ـ معهد المحاصيل الحقلية ـ مركز البحوث الزر اعية ـ الجيزة ـ مصر.

\section{ملخص}

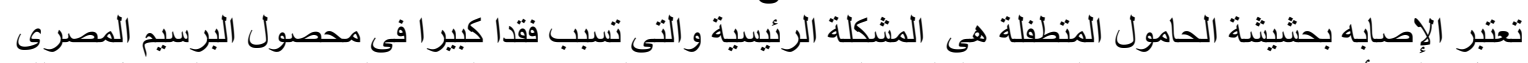

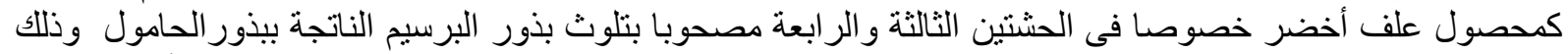

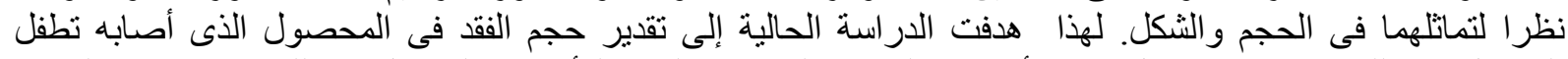

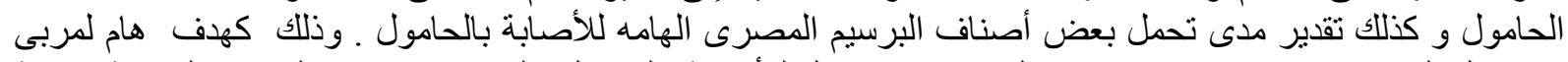

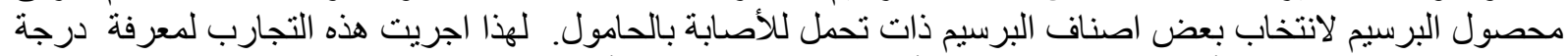

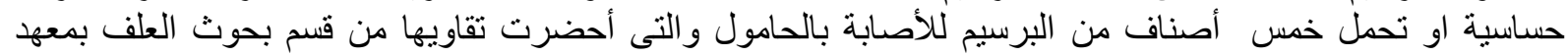

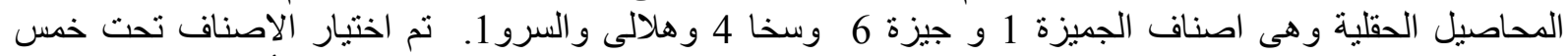

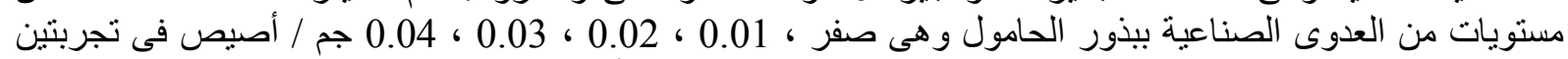

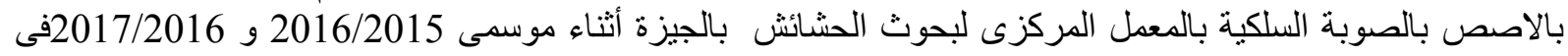

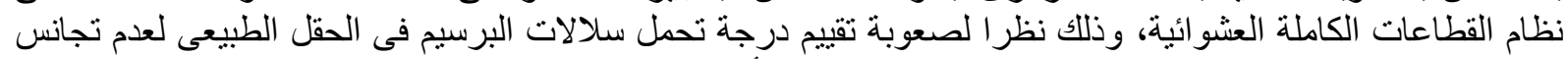

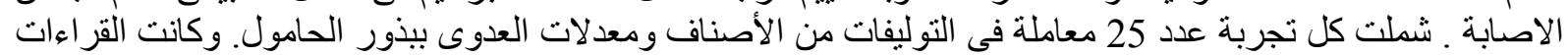

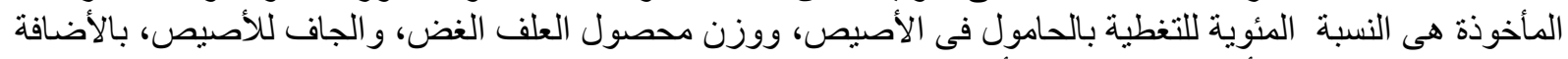

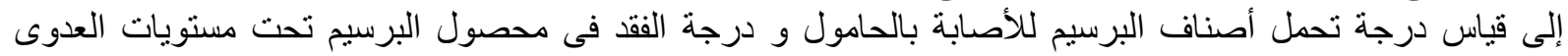

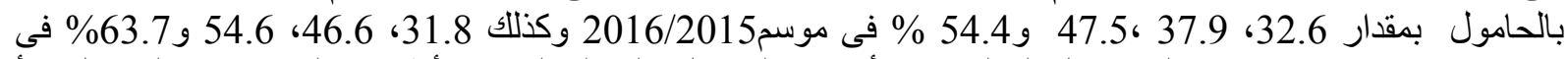

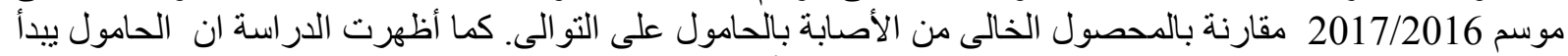

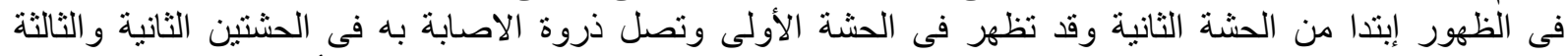

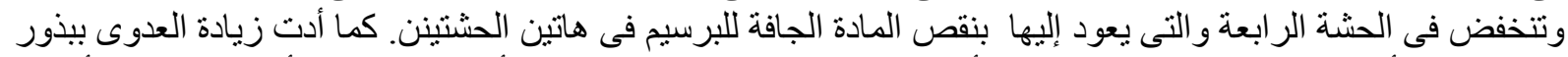

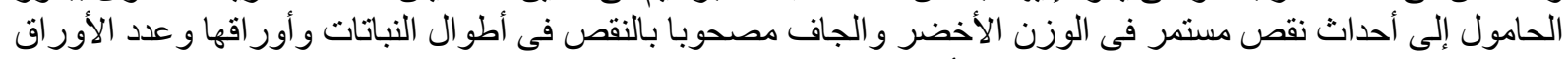

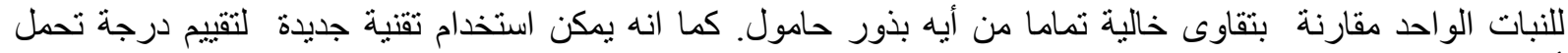

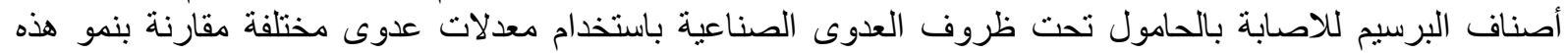

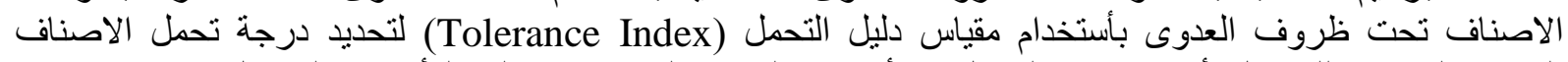

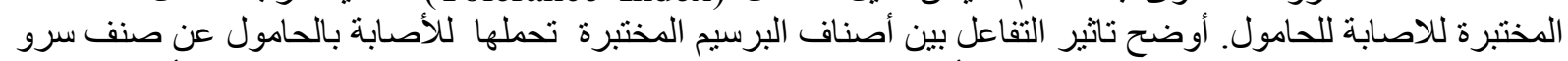

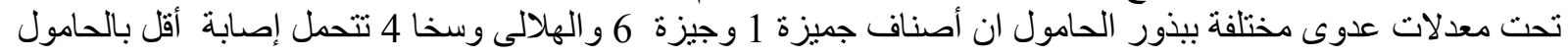

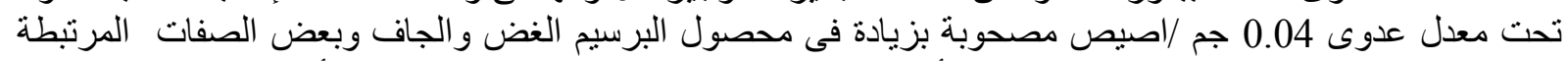

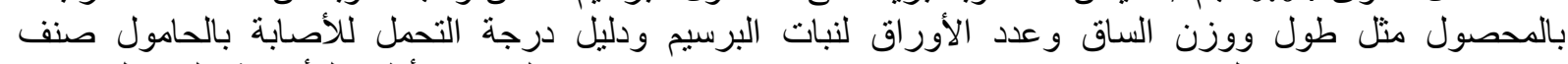

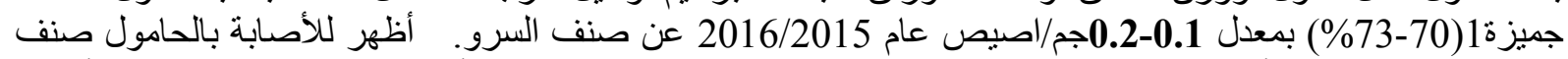

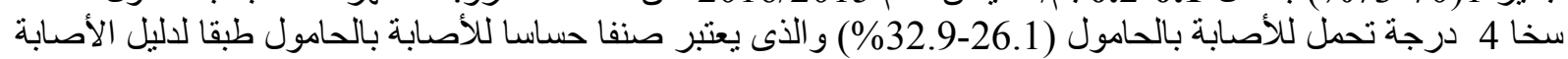

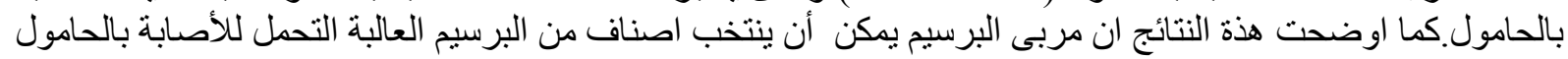

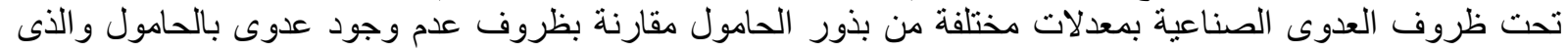

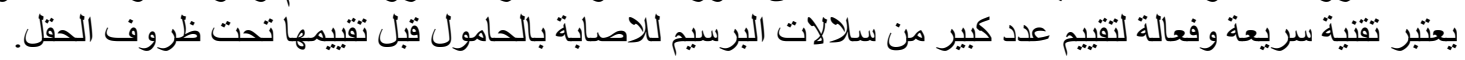

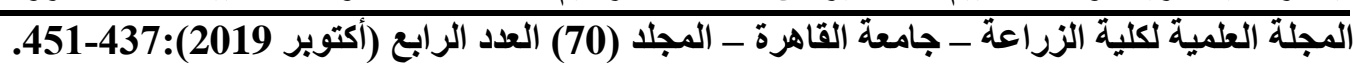

